\title{
Generalised hyperbolicity in spacetimes with string-like singularities
}

\author{
Yafet Sanchez Sanchez* \\ and James A. Vickers ${ }^{\dagger}$ \\ Mathematical Sciences and STAG Research Centre, \\ University of Southampton, \\ Southampton, \\ SO17 1BJ
}

August 30, 2016

\begin{abstract}
In this paper we present well-posedness results for $H^{1}$ solutions of the wave equation for spacetimes that contain string-like singularities. These results extend a framework in which one characterises gravitational singularities as obstruction to the dynamics of test fields rather than point particles. In particular, we discuss spacetimes with cosmic strings.
\end{abstract}

\section{Introduction}

The identification of the gravitational field with the spacetime background in General Relativity makes the domain of the solution of Einstein's equations not known a priori. This requires that one considers local solutions and then looks for suitable extensions. One of the issues that needs to be considered when extending the metric is the regularity of the metric. Since the Penrose and Hawking singularity theorems [1] the standard definition of a singularity has been in terms of geodesic incompleteness which intuitively can be thought of as an obstruction to the continuation of the world line of a free-falling observer. Such a definition requires the metric to be at least $C^{1,1}$ (i.e. the first derivatives of the metric are locally Lipschitz, denoted $C^{2-}$ in [1] for example) in order to guarantee the existence and uniqueness of geodesics. Furthermore, this regularity is the threshold where rough metrics and smooth metrics share the same causal structure [2, 3].

\footnotetext{
*E-mail:Y.SanchezSanchez@soton.ac.uk

${ }^{\dagger}$ E-mail:J.A.Vickers@soton.ac.uk
} 
However, if we consider Einstein's field equations simply as a hyperbolic evolution system, solutions can be obtained with the metric in Sobolev spaces which are compatible with regularity below $C^{1,1}[4$. Moreover, it has recently been shown that local well-posedness follows from having enough control over the $L^{2}$ norm of the curvature on the spatial foliation and the radius of injectivity [5]. From this point of view, the relevant condition to ensure the well-posedness of the evolution equations is not a pointwise condition on the curvature, but rather an $L^{2}$ type condition on the metric and its derivatives. In this context, an alternative is to consider a singularity as an obstruction to the evolution of a test-field rather than as an obstruction to the evolution of a particle, along a causal geodesic. This point of view was called generalised hyperbolicity by Clarke [6], and involves regarding certain traditional singularities as interior points in a spacetime with low regularity and then proving local well-posedness of the wave equation in the rough extension. The closely related concept of wave-regularity was introduced by Ishibashi and Hosoya [7] in their study of the wave equation in static singular spacetimes with timelike singularities. They used this term to define the well-posedness of the wave equation in the sense that unique solutions exist and there is no freedom in the boundary conditions one imposes on the singularity. A full discussion on this and related concepts can be found in $[8,9$.

In previous work we looked at the concept of wave-regularity for curveintegrable spacetimes [8] and spacetimes with singular hypersurfaces [9]. In this paper we extend these results to spacetimes with string like singularities. These are timelike singularities of co-dimension 2. As explained below, a natural condition is to require the solutions to lie in the Sobolev space $H^{1}$ (which requires the solutions and their first derivatives to be square integrable) and for clarity we now state precisely what we mean by $H^{1}$-wave regularity:

Definition 1 A point $p$ in $\left(M, g_{a b}\right)$ is $H^{1}$ wave regular if there is a region, $\Sigma_{(0, T]}=\Sigma \times(0, T]$, where $\Sigma$ is an open bounded region of an $n$-dimensional manifold containing $p$, such that the initial value problem on the initial hypersurface $\Sigma_{0}=\Sigma \times\{0\}$ for the wave equation $\square_{g} u=f$ on $\Sigma_{(0, T]}$ is locally well posed in the following sense:

- There exists a solution in the function space $H^{1}\left(\Sigma_{(0, T]}\right)$

- The solution is unique in the function space $H^{1}\left(\Sigma_{(0, T]}\right)$

- The solutions in the space $H^{1}\left(\Sigma_{(0, T]}\right)$ are stable with respect to initial data.

A point $p$ is weakly wave regular if it only satisfies the first two conditions. A strongly wave regular spacetime is defined to be one such that every point $p$ in $\left(\mathcal{M}, g_{a b}\right)$ is strongly wave regular. 
Notice that, if a solution $u$ is in $H^{1}$ and the components of the metric are bounded, then the energy momentum is integrable and satisfies $T^{a b}[u] \in L^{1}\left(\Sigma_{(0, T]}\right)$ and can be defined as a distribution. Therefore, for locally bounded metrics, $H^{1}$-wave regularity implies the integrability of the energy momentum tensor of the solutions. Moreover, canonical quantisation schemes of free scalar fields require the existence of symplectic structures formed by products of the solution and its derivatives [10]. In general, these structures will be ill-defined for solutions with less regularity. This is particularly problematic if one expects to be able to take into account the quantum behaviour of the field. Additionally, if there do not exist $H^{1}$ solutions to the scalar wave equation there will not exist $H^{1}$ solutions to the linearised Einstein equations.

In this paper, we provide tools to establish the existence and uniqueness of solutions of the wave equation with $H^{1}$-regularity in singular spacetimes. We also give examples of how $H^{1}$-wave regularity can be applied to a number of physically important scenarios. In $\S 2$ we prove a general theorem for wave equations in rough backgrounds with metrics that satisfy certain conditions (see geometric conditions 1 in $\S 2.1$ ) which are satisfied by cosmic string type singularities for example. The basic proof of the theorem follows the method of Evans [11, §7.2] and uses Galerkin approximation methods together with energy estimates for the wave operator. The method of proof is different from that used in [9] and reflects real differences in the type of singularity under consideration. For hypersurface singularities we were able to obtain energy estimates for the first order system and its adjoint, and use these to obtain existence using the Hahn-Banach theorem. For the string type singularities under consideration here, the special form of the metric means that we have good energy estimates for the wave operator but not for its adjoint in $H^{1}$. On the other hand, the time derivatives of the metric coefficients are well-behaved which is crucial to the use of a Galerkin approximation. This allows us to prove existence, uniqueness and stability of weak solutions with the required regularity. However the results differ from those in [11] in that we explicitly lower the differentiability (although see [12]), generalise the results to curved spacetimes with a special emphasis on the $n+1$ decomposition of spacetime and use a different method of proof to establish uniqueness and stability which allows the result to be generalised if one works with more general gauges. Furthermore, we show that under the geometric conditions 1 and the hypothesis of lemma 2 the energy momentum is not only integrable in spacetime, but can also be defined distributionally on any constant time hypersurface. In $\S 3$ we discuss how our theorems apply to a large class of spacetimes with cosmic string type singularities and show that, from this perspective, cosmic string singularities should not be regarded as strong gravitational singularities, even though the curvature is not locally bounded, nor even in some cases even locally integrable. Finally at the end of the section, we discuss the relationship of our approach to 
other work and the importance of low regularity solutions for any discussion of the Strong Cosmic Censorship Conjecture.

Notation. When considering the details of the function spaces $L^{2}, H^{1}$ etc. we first write the domain and then the measure considered, e.g., $L^{2}\left(\Sigma, \nu_{h}\right)$. When the measure is the one associated to the volume form $d x^{n}$, (or $d x^{n+1}$ when the domain is the whole spacetime) we will omit the measure and just write $L^{2}(\Sigma)$. The time dependence of functions will be explicitly stated at the beginning of the theorems. However, where there is no risk of confusion, the time dependence will not be made explicit in calculations and estimates. We also denote the derivative of a function $u$ with respect $t$ by $u_{t}$ and by $u_{i}$ if it is with respect to the other $x^{i}$-coordinates. When a function $d(t)$ depends only on time we denote the derivative by $\dot{d}(t)$. Additionally to avoid cumbersome notation we will not always explicitly use $\sum$ to denote a sum and we use instead Einstein's summation notation, with roman letters $a, b \ldots$ etc used for summations over $0 \cdots n$ and $i, j \ldots$ etc used for summations over $1 \cdots n$. We take the signature of the metric to be $(+,-,-, \ldots,-)$.

\section{The main theorem}

\subsection{The general setting}

Let $\Sigma_{(0, T]}=\Sigma \times(0, T]$ be a $(n+1)$-dimensional domain equipped with a Lorentzian metric $g_{a b}$, where $\Sigma$ is an open bounded region of a $n$-dimensional manifold. Now using a $n+1$ decomposition of spacetime the line element of the metric may be written in the form:

$$
d s^{2}=+N^{2} d t^{2}-\gamma_{i j}\left(d x^{i}+\beta^{i} d t\right)\left(d x^{j}+\beta^{j} d t\right)
$$

where $N$ is the lapse function, $\beta^{i}$ is the shift and $\gamma_{i j}$ is the induced metric on $\Sigma$.

The class of metrics we are going to consider requires that there is a foliation of the domain $\Sigma_{(0, T]}$ and suitable coordinates $\left(t, x^{i}\right)$ such that the following conditions hold.

\section{Geometric Conditions 1 .}

1. $\gamma^{i j} \in C^{1}\left([0, T], L^{\infty}(\Sigma)\right)$ i.e. $t \mapsto \gamma^{i j}(t)$ is a differentiable map into the space of locally bounded 3-metrics.

2. The volume form given by $\sqrt{\gamma}$, for the induced metric $\gamma_{i j}$ is bounded from below by a positive real number, i.e., $|\sqrt{\gamma}|>\eta$ for $\eta \in \mathbb{R}^{+}$

3. The lapse function $N$ can be chosen as $N=\sqrt{\gamma}$

4. The shift can be chosen in such a way that $\beta^{i}=0$ 
5. There exist a constant $\theta>0$ such that

$$
\sum_{i, j=0}^{n} \gamma^{i j} \gamma \xi_{i} \xi_{j} \geqslant \theta|\xi|^{2}
$$

for all $(t, x) \in \Sigma_{(0, T]}, \xi \in \mathbb{R}^{n}$

Condition 3. on the lapse function can be weakened to require only that it is a bounded function with a positive lower bound i.e.,

$3^{\prime}$. The lapse function $N$ is $C^{1}\left((0, T], L^{\infty}(\Sigma)\right)$ and $|N|>\omega$ for $\omega \in \mathbb{R}^{+}$

However this is at the expense of adding linear terms in time and to avoid undue complications in the formulae we do not pursue this here. Note however the method of proof in $\$ 2.3 .2$ has been modified from that in Evans [11] to allow for this possibility.

We want to obtain weak solutions to the following initial/boundary problem for the wave equation:

$$
\begin{aligned}
\square_{g} u & =f \text { in } \Sigma_{(0, T]} \\
u & =0 \text { on } \partial \Sigma \times[0, T] \\
u(0, x) & =u_{0} \text { on } \Sigma_{0}=\Sigma \times\{t=0\} \\
u_{t}(0, x) & =h \text { on } \Sigma_{0}=\Sigma \times\{t=0\}
\end{aligned}
$$

where $f: \Sigma_{(0, T]} \rightarrow \mathbb{R}$ is a given source and $u_{0}: \Sigma \rightarrow \mathbb{R}, h: \Sigma \rightarrow \mathbb{R}$ are given initial conditions.

For a metric with a general $n+1$ splitting given by (1) the wave operator is given by:

$$
\begin{array}{r}
\square_{g} u=\frac{1}{N \sqrt{\gamma}}\left(\partial_{t}\left(N \sqrt{\gamma} \frac{1}{N^{2}} \partial_{t} u\right)\right) \\
+\frac{1}{N \sqrt{\gamma}}\left(\partial_{t}\left(N \sqrt{\gamma} \frac{\beta^{i}}{N^{2}} \partial_{i} u\right)+\partial_{j}\left(N \sqrt{\gamma} \frac{\beta^{j}}{N^{2}} \partial_{t} u\right)\right) \\
-\frac{1}{N \sqrt{\gamma}} \partial_{i}\left(N \sqrt{\gamma}\left(\gamma^{i j}-\frac{\beta^{i} \beta^{j}}{N^{2}}\right) \partial_{j} u\right)
\end{array}
$$

Taking into account the geometric conditions 1 we obtain

$$
\square_{g} u=\frac{u_{t t}}{\gamma}-\frac{L u}{\gamma}
$$

where $-L$ is an elliptic operator in divergence form given by:

$$
-L u=-\left(\gamma^{i j} \gamma u_{j}\right)_{i}
$$


Notice that the geometric conditions imply that $L$ is a uniformly elliptic operator.

We can associate to the operator $-L$ the bilinear form given by:

$$
B[u, v ; t]:=\int_{\Sigma} \gamma^{i j}(t, x) \gamma(t, x) u_{i} v_{j} d x^{n}
$$

Definition 2 We say a function:

$$
u \in L^{2}\left(0, T ; H_{0}^{1}(\Sigma)\right), \text { with } u_{t} \in L^{2}\left(0, T ; L^{2}(\Sigma)\right), u_{t t} \in L^{2}\left(0, T ; H^{-1}(\Sigma)\right)
$$

is a local weak solution of the hyperbolic initial/boundary problem (2) provided that locally:

1. For each $v \in L^{2}\left(0, T ; H_{0}^{1}(\Sigma)\right)$,

$$
\int_{0}^{T}<u_{t t}, v>d t+\int_{0}^{T} B[u, v ; t] d t=(f, v)_{L^{2}\left(\Sigma_{(0, T]}, \mu_{g}\right)}
$$

where $\mu_{g}=\sqrt{-g} d^{n+1} x=\gamma d^{n+1} x$ and $<\cdot, \cdot>$ denotes the dual pairing between the $H^{-1}(\Sigma)$ and $H_{0}^{1}(\Sigma)$ Sobolev spaces.

2. $u(0, x)=u_{0}(x), u_{t}(0, x)=h(x)$ where $u_{0} \in H_{0}^{1}\left(\Sigma_{0}\right)$ and $h \in L^{2}\left(\Sigma_{0}\right)$

We motivate definition 2 by the following calculation. For the moment assume the metric and the solution are smooth and that $\square_{g} u=f$. Multiplying by an element $v \in L^{2}\left(0, T ; H_{0}^{1}(\Sigma)\right)$ and integrating we obtain:

$$
\begin{aligned}
\int_{\Sigma_{(0, T]}} f v \mu_{g} & =\int_{\Sigma_{(0, T]}}\left(\square_{g} u\right) v \mu_{g} \\
& =\int_{\Sigma_{(0, T]}}\left(\frac{1}{\gamma} u_{t t}-\frac{1}{\gamma} L u\right) v \gamma d^{n+1} x \\
& =\int_{0}^{T} \int_{\Sigma}\left(u_{t t}-L u\right) v d^{n} x d t \\
& =\int_{0}^{T} \int_{\Sigma}\left(u_{t t} v\right) d^{n} x d t-\int_{0}^{T} \int_{\Sigma}\left(\gamma^{i j} \gamma u_{j}\right)_{i} v d^{n} x d t \\
& =\int_{0}^{T} \int_{\Sigma}\left(u_{t t} v\right) d^{n} x d t+\int_{0}^{T} \int_{\Sigma} \gamma^{i j} \gamma u_{j} v_{i} d^{n} x d t \\
& =\int_{0}^{T}<u_{t t}, v>d t+\int_{0}^{T} B[u, v ; t] d t
\end{aligned}
$$

The final equation is the definition of a weak solution provided the regularity of the solution and the metric allows the integral to be well defined. This is indeed the case given the geometric conditions 1. The Sobolev embedding theorem in one dimension implies that $u \in C\left([0, T], L^{2}(\Sigma)\right) \cap$ $C^{1}\left([0, T], H^{-1}(\Sigma)\right)$ and therefore condition 2 makes sense. 


\subsection{Main result}

The main result we prove can be formally stated as follows:

Theorem 1 (Well-posedness in $\left.H^{1}\right)$ Let $\left(\Sigma_{(0, T]}, g_{a b}\right)$ be a region of a spacetime satisfying the geometric conditions 1 . Then the region $\Sigma_{(0, T]}$ is wave-regular. That is the wave equation is well-posed in the following sense: Given $\left(u_{0}, h\right) \in H_{0}^{1}\left(\Sigma_{0}\right) \times L^{2}\left(\Sigma_{0}\right)$ there exists a unique weak solution $u$ in $H^{1}\left(\Sigma_{(0, T]}\right)$ of $\square_{g} u=f$ in the sense of definition 2 with initial conditions

1. $u(0, \cdot)=\left.u\right|_{\Sigma_{0}}=u_{0}$

2. $u_{t}(0, \cdot)=\left.\frac{\partial u}{\partial t}\right|_{\Sigma_{0}}=h$

that is stable with respect to initial data in $H_{0}^{1}\left(\Sigma_{0}\right) \times L^{2}\left(\Sigma_{0}\right)$. Moreover, under the hypothesis of Lemma 2 the components of the energy momentum tensor associated with the solution satisfy $T^{a b}[u] \in C^{0}\left([0, T], L^{1}(\Sigma)\right)$.

\subsection{Existence of solutions}

To prove existence of solutions we employ Galerkin's method. This requires several steps. We begin by showing uniqueness and existence of approximate solutions, then we establish a uniform estimate for the solutions and finally we take a limit in a proper weak topology which converges to the required weak solution.

We start by choosing smooth functions $w_{k}(x)$ such that:

$$
\begin{gathered}
\left\{w_{k}\right\}_{k=1}^{\infty} \text { is an orthogonal basis of } H_{0}^{1}(\Sigma) \\
\left\{w_{k}\right\}_{k=1}^{\infty} \text { is an orthonormal basis of } L^{2}(\Sigma)
\end{gathered}
$$

We can form the desired basis by choosing the eigenvectors of the Laplace operator $\Delta$ in the given local coordinates [11].

Now fix a positive integer $m$, write

$$
u^{m}(t, x):=\sum_{k=1}^{m} d_{m}^{k}(t) w_{k}(x)
$$

and consider for each $k=1, \ldots, m$ the equation:

$$
\left(u_{t t}^{m}, w_{k}\right)_{L^{2}(\Sigma)}+B\left[u^{m}, w_{k} ; t\right]=\left(f, w_{k}\right)_{L^{2}\left(\Sigma, N \nu_{\gamma}\right)}
$$

where $\nu_{\gamma}$ is the volume form associated to the induced metric $\gamma_{i j}$ on $\Sigma$. The system of equations (12) can be arranged as a system of linear ODE's given by

$$
\ddot{d}_{m}^{k}(t)+\sum_{l=1}^{m} e^{k l}(t) d_{m}^{l}(t)=f^{k}(t)
$$


where $e^{k l}(t):=B\left[w_{l}, w_{k} ; t\right]=\int_{\Sigma} \gamma^{i j}(t, x) \gamma(t, x) w_{l_{i}} w_{k_{j}} d x^{n}$ and $f^{k}(t):=$ $\left(f, w_{k}\right)_{L^{2}\left(\Sigma, N \nu_{\gamma}\right)}$ for each $k=1, \ldots, m$.

We also require that the system satisfies the initial conditions

$$
d_{m}^{k}(0)=\left(u_{0}, w_{k}\right)_{L^{2}\left(\Sigma_{0}\right)}, \quad \dot{d}_{m}^{k}(0)=\left(h, w_{k}\right)_{L^{2}\left(\Sigma_{0}\right)}
$$

for $k=1, \ldots, m$.

The functions $e^{k l}(t)$ are continuous in $t$ as $\gamma^{i j}(t, x) \gamma(t, x) \in C^{1}\left([0, T], L^{\infty}(\Sigma)\right)$. Then by standard local existence and uniqueness theorems for linear ordinary differential equations we obtain a unique $d_{m}^{k}(t) \in C^{2}([0, T])$ for every $k=1, \ldots, m$.

Therefore we have shown that for each $m$ there is a unique solution, $u^{m}$, satisfying (12) and (14) which we call the m-approximate solution.

\subsubsection{Energy estimates}

In this section we establish the following energy estimate.

Theorem 2 There exists a constant $C$, depending only on $\Sigma, T$ and the coefficients of $L$ such that

$$
\begin{array}{r}
\max _{t \in(0, T]}\left(\left\|u^{m}(t, \cdot)\right\|_{H_{0}^{1}(\Sigma)}+\left\|u_{t}^{m}(t, \cdot)\right\|_{L^{2}(\Sigma)}+\left\|u_{t t}^{m}\right\|_{L^{2}\left([0, T] ; H^{-1}(\Sigma)\right)}\right) \\
\leqslant C\left(\|f\|_{L^{2}\left([0, T] ; L^{2}\left(\Sigma, N \nu_{\gamma}\right)\right)}+\left\|u_{0}\right\|_{H_{0}^{1}(\Sigma)}+\|h\|_{L^{2}(\Sigma)}\right)
\end{array}
$$

We start by multiplying equality $(12)$ by $\dot{d}_{m}^{k}(t)$, sum from $k=1, \ldots, m$ and use (11) to obtain

$$
\left(u_{t t}^{m}, u_{t}^{m}\right)_{L^{2}(\Sigma)}+B\left[u^{m}, u_{t}^{m} ; t\right]=\left(f, u_{t}^{m}\right)_{L^{2}\left(\Sigma, N \nu_{\gamma}\right)}
$$

Using the fact that that

$$
\left(u_{t t}^{m}, u_{t}^{m}\right)_{L^{2}(\Sigma)}=\frac{1}{2} \frac{d}{d t}\left\|u_{t}^{m}\right\|_{L^{2}(\Sigma)}^{2}
$$

and that

$$
B\left[u^{m}, u_{t}^{m} ; t\right]=\frac{d}{d t}\left(\frac{1}{2} B\left[u^{m}, u^{m} ; t\right]\right)-\frac{1}{2} \int_{\Sigma}\left(\gamma^{i j}(t, x) \gamma(t, x)\right)_{t} u_{i}^{m} u_{j}^{m}
$$

we have

$$
B\left[u^{m}, u_{t}^{m} ; t\right] \geqslant \frac{d}{d t}\left(\frac{1}{2} B\left[u^{m}, u^{m} ; t\right]\right)-C_{1}\left\|u^{m}\right\|_{H_{0}^{1}(\Sigma)}^{2}
$$

Combining equations (16), (17), (18) and (19) we obtain 


$$
\begin{aligned}
& \frac{d}{d t}\left(\left\|u_{t}^{m}\right\|_{L^{2}(\Sigma)}^{2}+B\left[u^{m}, u^{m} ; t\right]\right) \\
\leqslant & C_{2}\left(\left\|u_{t}^{m}\right\|_{L^{2}(\Sigma)}^{2}+\left\|u^{m}\right\|_{H_{0}^{1}(\Sigma)}^{2}+\|f\|_{L^{2}\left(\Sigma, N \nu_{\gamma}\right)}^{2}\right) \\
\leqslant & C_{3}\left(\left\|u_{t}^{m}\right\|_{L^{2}(\Sigma)}^{2}+B\left[u^{m}, u^{m} ; t\right]+\|f\|_{L^{2}\left(\Sigma, N \nu_{\gamma}\right)}^{2}\right)
\end{aligned}
$$

Where we have applied the uniform ellipticity condition in order to use the inequality

$$
\theta \int_{\Sigma}\left|\delta^{i j} u_{i}^{m} u_{j}^{m}\right| \leqslant B\left[u^{m}, u^{m} ; t\right]
$$

If we now define the "energy" $E(t)$ of a solution by:

$$
E(t)=\left\|u_{t}^{m}(t, \cdot)\right\|_{L^{2}(\Sigma)}^{2}+B\left[u^{m}, u^{m} ; t\right]
$$

Then inequality 22 reads

$$
\frac{d}{d t} E(t) \leqslant C_{3} E(t)+C_{3}\|f(t, \cdot)\|_{L^{2}\left(\Sigma, N \nu_{\gamma}\right)}^{2}
$$

and an application of Gronwall's inequality gives the estimate

$$
E(t) \leqslant e^{C_{4} t}\left(E(0)+C_{3} \int_{0}^{t}\|f(t, \cdot)\|_{L^{2}\left(\Sigma, N \nu_{\gamma}\right)}^{2}\right)
$$

However, we also have

$$
E(0) \leqslant C_{5}\left(\left\|u_{0}\right\|_{H_{0}^{1}(\Sigma)}^{2}+\|h\|_{L^{2}(\Sigma)}^{2}\right)
$$

which follows from the initial conditions for the approximate solutions together with $\left\|u^{m}(0)\right\|_{H_{0}^{1}(\Sigma)}^{2} \leqslant\left\|u_{0}\right\|_{H_{0}^{1}(\Sigma)}^{2},\left\|u_{t}^{m}(0)\right\|_{L^{2}(\Sigma)}^{2} \leqslant\|h\|_{L^{2}(\Sigma)}^{2}$.

Thus we obtain

$$
\begin{array}{r}
\max _{t \in(0, T]}\left(\left\|u_{t}^{m}\right\|_{L^{2}(\Sigma)}^{2}+B\left[u^{m}, u^{m} ; t\right]\right) \\
\leqslant C_{6}\left(\|f\|_{L^{2}\left([0, T] ; L^{2}\left(\Sigma, N \nu_{\gamma}\right)\right)}^{2}+\left\|u_{0}\right\|_{H_{0}^{1}(\Sigma)}^{2}+\|h\|_{L^{2}(\Sigma)}^{2}\right)
\end{array}
$$

Now we have from equation 12 that

$$
\begin{array}{r}
\left(u_{t t}^{m}, w_{k}\right)_{L^{2}(\Sigma)}=-B\left[u^{m}, w_{k} ; t\right]+\left(f, w_{k}\right)_{L^{2}\left(\Sigma, N \nu_{\gamma}\right)} \\
\leqslant C_{7}\left(\left\|u^{m}\right\|_{H_{0}^{1}(\Sigma)}+\|f\|_{L^{2}(\Sigma)}^{2}\right)\left\|w_{k}\right\|_{H_{0}^{1}(\Sigma)}
\end{array}
$$

where we have used the bounds on $N, \sqrt{\gamma}$ given by the geometric condition 1 and the Cauchy-Schwartz inequality. 
Since $\left(u_{t t}^{m}, w_{k}\right)=0$ for $k>m$ by construction, we have proved that

$$
\begin{aligned}
\left\|u_{t t}^{m}\right\|_{H^{-1}(\Sigma)} & =\sup _{v \in \operatorname{span}\left\{w_{k}\right\}} \frac{\left(u_{t t}^{m}, v\right)_{L^{2}(\Sigma)}}{\|v\|_{H_{0}^{1}(\Sigma)}} \\
& \leqslant C_{8} \sup _{v \in \operatorname{span}\left\{w_{k}\right\}} \frac{\left|(f, v)_{L^{2}(\Sigma)}\right|+\left|B\left(u^{m}, v ; t\right)\right|}{\|v\|_{H_{0}^{1}(\Sigma)}} \\
& \leqslant C_{9}\left(\|f\|_{\left.L^{2}(\Sigma)\right)}+\left\|u^{m}\right\|_{H_{0}^{1}(\Sigma)}\right)
\end{aligned}
$$

Squaring the above inequality, integrating in time and using equation 28 we obtain

$$
\int_{0}^{T}\left\|u_{t t}\right\|_{H^{-1}(\Sigma)}^{2} d t \leqslant C_{12}\left(\|f\|_{L^{2}\left([0, T] ; L^{2}\left(\Sigma, N \nu_{\gamma}\right)\right)}^{2}+\left\|u_{0}\right\|_{H_{0}^{1}(\Sigma)}^{2}+\|h\|_{L^{2}(\Sigma)}^{2}\right)
$$

which concludes the proof.

\subsubsection{Convergence to solutions}

We have shown that $\left\{u^{m}\right\}_{m=1}^{\infty}$ is bounded in $L^{2}\left(0, T ; H_{0}^{1}(\Sigma)\right),\left\{u_{t}^{m}\right\}_{m=1}^{\infty}$ is bounded in $L^{2}\left(0, T ; L^{2}(\Sigma)\right)$ and $\left\{u_{t t}^{m}\right\}_{m=1}^{\infty}$ is bounded in $L^{2}\left(0, T ; H^{-1}(\Sigma)\right)$ We now make use of the following Theorem [11]

Theorem 3 Let $X$ be a reflexive Banach space and suppose the sequence $\left\{u_{k}\right\}_{k=1}^{\infty} \subset X$ is bounded. Then there exist a sub-sequence $\left\{u_{k_{j}}\right\}_{j=1}^{\infty} \subset$ $\left\{u_{k}\right\}_{k=1}^{\infty}$ and $u \in X$ such that $u_{k_{j}} \rightarrow u$, i.e., $\left\{u_{k_{j}}\right\}$ converges weakly to $u$.

Using the theorem there exists a sub-sequence of approximate functions $\left\{u^{m_{l}}\right\}_{l=1}^{\infty}$ such that

- $u^{m_{l}} \rightarrow L^{2}\left(0, T ; H_{0}^{1}(\Sigma)\right)$

- $u_{t}^{m_{l}} \rightarrow L^{2}\left(0, T ; L^{2}(\Sigma)\right)$

- $u_{t t}^{m_{l}} \rightarrow L^{2}\left(0, T ; H^{-1}(\Sigma)\right)$

In order to show this is a weak solution we must now verify that the limit of the sequence satisfies conditions 1 and 2 of definition 2 .

To verify condition 1 we multiply $(12)$ by a function $\phi(t) \in C^{\infty}([0, T])$ and integrate with respect to time to give

$\int_{0}^{T}\left(\left(u_{t t}^{m_{l}}, \phi(t) w_{k}\right)_{L^{2}(\Sigma)}+B\left[u^{m_{l}}, \phi(t) w_{k} ; t\right]\right) d t=\int_{0}^{T}\left(f, \phi(t) w_{k}\right)_{L^{2}\left(\Sigma, N \nu_{\gamma}\right)} d t$ 
Then taking the limit as $m_{l} \rightarrow \infty$ we obtain

$$
\int_{0}^{T}\left(<u_{t t}, \phi(t) w_{k}>+B\left[u, \phi(t) w_{k} ; t\right]\right) d t=\int_{0}^{T}\left(f, \phi(t) w_{k}\right)_{L^{2}\left(\Sigma, N \nu_{\gamma}\right)} d t
$$

Thus for any test function of the form $v=\sum_{k=1}^{N} \phi^{k}(t) w_{k}(x)$ we have that equality (35) is satisfied. Moreover, test functions of that form are dense in $L^{2}\left(0, T ; H_{0}^{1}(\Sigma)\right)$. Therefore, we have shown that

$$
\int_{0}^{T}\left(<u_{t t}, v>+B[u, v ; t]\right) d t=\int_{0}^{T}(f, v)_{L^{2}\left(\Sigma, N \nu_{\gamma}\right)} d t=\int_{\Sigma_{(0, T]}} f v \mu_{g}
$$

for any $v \in L^{2}\left(0, T ; H_{0}^{1}(\Sigma)\right)$.

Finally we need to verify that the solution also satisfies the initial conditions. Using the initial condition (14 and the fact that $\left\{w_{k}\right\}$ is a basis of $L^{2}\left(\Sigma_{0}\right)$ we obtain

$$
\begin{aligned}
& u^{m_{l}}(0, \cdot) \rightarrow u_{0} \text { in } L^{2}\left(\Sigma_{0}\right) \\
& u_{t}^{m_{l}}(0, \cdot) \rightarrow h \text { in } L^{2}\left(\Sigma_{0}\right)
\end{aligned}
$$

Then using the fact that $L^{2}$ convergence of a sequence of functions to a function in $L^{2}$ implies there is a sub-sequence that converges a.e. pointwise we can find a sub-sequence $\left\{u^{m_{l_{r}}}(0, \cdot)\right\}_{r=1}^{\infty}$ such that it converges a.e. pointwise to $u_{0}$. Notice that then we can take a sub-sequence $\left\{u^{m_{l_{r}}}(0, \cdot)\right\}_{s=1}^{\infty}$ of the sub-sequence $\left\{u^{m_{l_{r}}}(0, \cdot)\right\}_{r=1}^{\infty}$ to add the a.e. pointwise convergence of the sequence $\left\{u_{t}^{m_{l_{s}}}(0, \cdot)\right\}_{s=1}^{\infty}$ to the function $h$.

Therefore, the limit $u^{m_{l_{r}}} \rightarrow u$ gives the desired weak solution.

\subsection{Uniqueness and stability with respect to the initial data}

The proof of uniqueness and stability relies on the energy estimate (15). By letting $m$ tend to infinity and using the fact that the norm is sequentially weakly lower-semicontinuous [11, we obtain the bound that the weak solution satisfies

$$
\begin{array}{r}
\max _{t \in(0, T]}\left(\|u(t, \cdot)\|_{H_{0}^{1}(\Sigma)}+\left\|u_{t}(t, \cdot)\right\|_{L^{2}(\Sigma)}\right) \\
\leqslant C\left(\|f\|_{L^{2}\left([0, T] ; L^{2}\left(\Sigma, N \nu_{\gamma}\right)\right)}+\left\|u_{0}\right\|_{H_{0}^{1}(\Sigma)}+\|h\|_{L^{2}(\Sigma)}\right)
\end{array}
$$

Therefore, if $u=u_{1}-u_{2}$ is the difference between two weak solutions satisfying the same initial conditions $u_{0}, h$ with the same source function $f$, then $u$ is a weak solution with vanishing initial data $u_{0}=h=0$ and source function $f=0$. 
Hence

$$
\left(\|u(t, \cdot)\|_{H_{0}^{1}(\Sigma)}+\left\|u_{t}(t, \cdot)\right\|_{L^{2}(\Sigma)}\right) \leqslant 0
$$

for all $0 \leqslant t \leqslant T$ which implies $u=0$ and therefore $u_{1}=u_{2}$.

We now prove the stability of the solution with respect to initial data. To make the concept precise we say that the solution $u$ is continuously stable in $H^{1}\left(\Sigma_{(0, T]}\right)$ with respect to initial data in $H_{0}^{1}\left(\Sigma_{0}\right) \times L^{2}\left(\Sigma_{0}\right)$, if given $\epsilon>0$ there is a $\delta$ depending on $u_{0}, h, f$ such that if:

$$
\left\|u_{0}-\tilde{u_{0}}\right\|_{H_{0}^{1}\left(\Sigma_{0}\right)} \leqslant \delta \text {, and }\|h-\tilde{h}\|_{L^{2}\left(\Sigma_{0}\right)} \leqslant \delta
$$

for $\left(u_{0}, h\right) \in H_{0}^{1}\left(\Sigma_{0}\right) \times L^{2}\left(\Sigma_{0}\right)$ and

$$
\|f-\tilde{f}\|_{L^{2}\left([0, T] ; L^{2}\left(\Sigma, N \nu_{\gamma}\right)\right)} \leqslant \delta
$$

for $f \in{L^{2}\left([0, T] ; L^{2}\left(\Sigma, N \nu_{\gamma}\right)\right)}$ then

$$
\|u-\tilde{u}\|_{H_{0}^{1}\left(\Sigma_{(0, T]}\right)} \leqslant \epsilon
$$

where $\tilde{u}$ is a solution with initial data given by $\left.\tilde{u}\right|_{\Sigma_{0}}=\tilde{u}_{0}$ and $\left.\tilde{u}_{t}\right|_{\Sigma_{0}}=\tilde{h}$ with source function $\tilde{f}$.

Now squaring $(39)$ and integrating in time from 0 to $\tau \leqslant T$ we have:

$$
\begin{aligned}
\left(\|u-\tilde{u}\|_{\Sigma_{(0, T]}}\right)^{2} & \leqslant \int_{0}^{\tau}\left(\|u(t, \cdot)\|_{H_{0}^{1}(\Sigma)}^{2}+\left\|u_{t}(t, \cdot)\right\|_{L^{2}(\Sigma)}^{2}\right) d t \\
& \leqslant K \int_{0}^{\tau}\left(\left\|u_{0}-\tilde{u}_{0}\right\|_{H_{0}^{1}(\Sigma)}^{2}+\|h-\tilde{h}\|_{L^{2}(\Sigma)}^{2}\right)+\|f-\tilde{f}\|_{L^{2}\left([0, T] ; L^{2}\left(\Sigma, N \nu_{\gamma}\right)\right)} d t
\end{aligned}
$$

for $\tau \leqslant T$ and with $K$ a suitable constant.

Now choosing $\delta=\frac{\epsilon}{\sqrt{3 \tau C}}$ we obtain the inequality:

$$
\left(\|u-\tilde{u}\|_{\Sigma_{(0, T]}}\right)^{2} \leqslant K \int_{0}^{\tau} \frac{2 \epsilon^{2}}{3 \tau K}+\frac{\epsilon^{2}}{3 \tau K} d t \leqslant \epsilon^{2}
$$

which establishes stability with respect to the initial data.

\subsection{Integrability of the energy momentum tensor}

The regularity of the solutions allows us to make sense of the energy momentum tensor of the scalar field $u$ as a tensor with $L^{1}\left(\Sigma_{(0, T]}\right)$ components given by

$$
T^{a b}[u]=\left(g^{a c} g^{b d}-\frac{1}{2} g^{a b} g^{c d}\right) u_{c} u_{d}-\frac{1}{2} g^{a b} u^{2}
$$

We now show that $T^{a b}[u](t, \cdot)$ is in $L^{1}\left(\Sigma_{t}\right)$ for all $0 \leqslant t \leqslant T$. To prove this, notice that it is enough to show that $u \in C\left([0, T] ; H_{0}^{1}(\Sigma)\right) \cap C^{1}\left(0, T ; L^{2}(\Sigma)\right)$. This result also allows us to establish the existence and uniqueness of solutions in $\Sigma_{(0, T]}$ given initial data on any hypersurface $\Sigma_{t}$ with $0 \leqslant t \leqslant T$. In this section we closely follow the exposition given in [12]. 
Proposition 1 Let $u$ be a weak solution as defined in Definition 2 with $f \in L^{2}\left(\Sigma_{(0, T]}\right)$ and initial data $\left(u_{0}, h\right) \in H_{0}^{1}\left(\Sigma_{0}\right) \times L^{2}\left(\Sigma_{0}\right)$. Additionally let the metric satisfies the geometric condition 1 and the hypothesis of Lemma 2. Then $u \in C\left([0, T] ; H_{0}^{1}(\Sigma)\right) \cap C^{1}\left(0, T ; L^{2}(\Sigma)\right)$

To prove this proposition, we use the following Lemmas

Lemma 1 Suppose that $V, H$ are Hilbert spaces and $V \hookrightarrow H$ is densely and continuously embedded in $H$. If

$$
u \in L^{\infty}(0, T ; V), u_{t} \in L^{2}(0, T ; H),
$$

then $u \in C_{w}([0, T] ; V)$ is weakly continuous.

Then from the fact that $H_{0}^{1} \hookrightarrow L^{2} \hookrightarrow H^{-1}$ and the energy estimate we have that $u \in C_{w}\left([0, T] ; H_{0}^{1}(\Sigma)\right)$ and $u_{t} \in C_{w}\left([0, T] ; L^{2}(\Sigma)\right)$

Lemma 2 Let $u$ be a weak solution that satisfies $u_{t t}+L u \in L^{2}\left(0, T ; L^{2}(\Sigma)\right)$ and assume there is a mollified sequence of functions given by

$$
\left\{u^{\epsilon}(t, x)=\eta^{\epsilon}(t) *(\xi u(t, x))\right\}
$$

where $\left\{\eta^{\epsilon}(t)\right\}_{\epsilon}$ is a strict delta net and $\xi$ is a smooth cut-off function vanishing outside the interval $(0, T)$ and equal to one in some sub-interval $I \subset(0, T)$ such that $u_{t t}^{\epsilon}+L u^{\epsilon} \in L^{2}\left(0, T ; L^{2}(\Sigma)\right)$ and in the limit $\epsilon \rightarrow 0$ we have $u_{t t}^{\epsilon}+L u^{\epsilon} \rightarrow u_{t t}+L u$ in $L^{2}\left(0, T ; L^{2}(\Sigma)\right)$

Then

$$
\frac{1}{2} \frac{d}{d t}\left(\left\|u_{t}\right\|_{L^{2}(\Sigma)}^{2}+B[u, u ; t]\right)=\left(u_{t t}+L u, u_{t}\right)_{L^{2}(\Sigma)}^{2}+\frac{1}{2} B_{t}[u, u ; t]
$$

where

$$
B_{t}[u, v ; t]:=\int_{\Sigma} \sum_{i, j=1}^{n}\left(\gamma^{i j}(t, x) N(t, x) \sqrt{\gamma(t, x)}\right)_{t} u_{x^{i}} v_{x^{j}} d x^{n}
$$

and

$$
E(t)=\left(\left\|u_{t}(t, \cdot)\right\|_{L^{2}(\Sigma)}^{2}+B[u, u ; t]\right):(0, T] \rightarrow \mathbb{R}
$$

is an absolutely continuous function.

Proof.

We have that

$$
\begin{gathered}
\frac{1}{2} \frac{d}{d t}\left(\left\|u_{t}^{\epsilon}\right\|_{L^{2}(\Sigma)}+B\left[u^{\epsilon}, u^{\epsilon} ; t\right]\right) \\
=\quad\left(u_{t t}^{\epsilon}, u_{t}^{\epsilon}\right)_{L^{2}(\Sigma)}^{2}+B\left[u^{\epsilon}, u_{t}^{\epsilon} ; t\right]+\frac{1}{2} B_{t}\left[u^{\epsilon}, u^{\epsilon} ; t\right] \\
=\quad\left(u_{t t}^{\epsilon}+L u^{\epsilon}, u_{t}^{\epsilon}\right)_{L^{2}(\Sigma)}+\frac{1}{2} B_{t}\left[u^{\epsilon}, u^{\epsilon} ; t\right]
\end{gathered}
$$


Taking the limit as $\epsilon \rightarrow 0$ give us the same result for the unmollified function $\xi u$ and hence equation (48) holds on every compact sub-interval of $(0, T)$. Now the derivative of the RHS of equation 50 is in $L^{1}(0, T)$ since using equation (51) and taking the limit $\epsilon \rightarrow 0$ we have

$$
\begin{aligned}
\int_{0}^{T}\left(u_{t t}+L u, u_{t}\right)_{L^{2}(\Sigma)} d t & \leqslant\left\|u_{t t}+L u\right\|_{L^{2}\left([0, T] ; L^{2}(\Sigma)\right)}\left\|u_{t}\right\|_{L^{2}\left([0, T] ; L^{2}(\Sigma)\right)} \\
& \leqslant\|f\|_{L^{2}\left([0, T] ; L^{2}(\Sigma)\right)}\|u\|_{L^{2}\left([0, T] ; L^{2}(\Sigma)\right)} \\
\int_{0}^{T} B_{t}[u, u ; t] & \leqslant C\|u\|_{L^{2}\left([0, T] ; H_{0}^{1}(\Sigma)\right)}^{2}
\end{aligned}
$$

Thus $E(t)$ is the integral of a $L^{1}$ function, so is absolutely continuous.

We can now prove Proposition 1 .

Proof of Proposition 1.

Using the weak continuity of $u_{t}$, the continuity of $E$ and the continuity of $a_{t}$ in $H_{0}^{1}$ we find that

$$
\begin{array}{r}
\left\|u_{t}(t, \cdot)-u_{t}\left(t_{0}, \cdot\right)\right\|_{L^{2}(\Sigma)}^{2}+B\left[u(t, \cdot)-u\left(t_{0}, \cdot\right), u(t, \cdot)-u\left(t_{0}, \cdot\right) ; t_{0}\right] \\
=\left\|u_{t}(t, \cdot)\right\|_{L^{2}(\Sigma)}^{2}+\left\|u_{t}\left(t_{0}, \cdot\right)\right\|_{L^{2}(\Sigma)}^{2} \\
+B\left[u(t, \cdot), u(t, \cdot) ; t_{0}\right]+B\left[u\left(t_{0}, \cdot\right), u\left(t_{0}, \cdot\right) ; t_{0}\right] \\
-2\left(u_{t}(t, \cdot), u_{t}\left(t_{0}, \cdot\right)\right)_{L^{2}(\Sigma)}-2 B\left[u(t, \cdot), u\left(t_{0}, \cdot\right) ; t_{0}\right] \\
=\left\|u_{t}(t, \cdot)\right\|_{L^{2}(\Sigma)}^{2}+B[u(t, \cdot), u(t, \cdot) ; t] \\
+\left\|u_{t}\left(t_{0}, \cdot\right)\right\|_{L^{2}(\Sigma)}^{2}+B\left[u\left(t_{0}, \cdot\right), u\left(t_{0}, \cdot\right) ; t_{0}\right] \\
-2 B\left[u(t, \cdot), u\left(t_{0}, \cdot\right) ; t_{0}\right]-2\left(u_{t}(t, \cdot), u_{t}\left(t_{0}, \cdot\right)\right)_{L^{2}(\Sigma)} \\
+B\left[u(t, \cdot), u(t, \cdot) ; t_{0}\right]-B[u(t, \cdot), u(t, \cdot) ; t] \\
=E(t)+E\left(t_{0}\right)+B\left[u(t, \cdot), u(t, \cdot) ; t_{0}\right]-B[u(t, \cdot), u(t, \cdot) ; t] \\
\left.-2\left(\left(u_{t}(t, \cdot), u_{t}\left(t_{0}, \cdot\right)\right)_{L^{2}(\Sigma)}+B\left[u(t, \cdot), u\left(t_{0}, \cdot\right) ; t_{0}\right]\right)\right)
\end{array}
$$

Therefore taking the limit $t \rightarrow t_{0}$ we obtain

$$
\begin{aligned}
& \lim _{t \rightarrow t_{0}}\left(\left\|u_{t}(t, \cdot)-u_{t}\left(t_{0}, \cdot\right)\right\|_{L^{2}(\Sigma)}^{2}+B\left[u(t, \cdot)-u\left(t_{0}, \cdot\right), u(t, \cdot)-u\left(t_{0}, \cdot\right) ; t_{0}\right]\right) \\
& =\lim _{t \rightarrow t_{0}} E(t)+E\left(t_{0}\right)+B\left[u(t, \cdot), u(t, \cdot) ; t_{0}\right]-B[u(t, \cdot), u(t, \cdot) ; t] \\
& \left.-2\left(\left(u_{t}(t, \cdot), u_{t}\left(t_{0}, \cdot\right)\right)_{L^{2}(\Sigma)}+B\left[u(t, \cdot), u\left(t_{0}, \cdot\right) ; t_{0}\right]\right)\right) \\
& =E\left(t_{0}\right)+E\left(t_{0}\right)-2\left(\left(u_{t}\left(t_{0}, \cdot\right), u_{t}\left(t_{0}, \cdot\right)+B\left[u\left(t_{0}, \cdot\right), u\left(t_{0}, \cdot\right) ; t_{0}\right]\right)\right) \\
& \left.=E\left(t_{0}\right)+E\left(t_{0}\right)-2\left(\left\|u_{t}\left(t_{0}, \cdot\right)\right\|_{L^{2}(\Sigma)}^{2}+B\left[u\left(t_{0}, \cdot\right), u\left(t_{0}, \cdot\right) ; t_{0}\right]\right)\right)=0
\end{aligned}
$$


Then using equation 23 we have

$$
\begin{gathered}
\lim _{t \rightarrow t_{0}}\left(\left\|u_{t}(t, \cdot)-u_{t}\left(t_{0}, \cdot\right)\right\|_{L^{2}(\Sigma)}^{2}+\theta\left\|u(t, \cdot)-u\left(t_{0}, \cdot\right)\right\|_{H_{0}^{1}(\Sigma)}^{2}\right) \\
\leqslant \lim _{t \rightarrow t_{0}}\left(\left\|u_{t}(t, \cdot)-u_{t}\left(t_{0}, \cdot\right)\right\|_{L^{2}(\Sigma)}^{2}+B\left[u(t, \cdot)-u\left(t_{0}, \cdot\right), u(t, \cdot)-u\left(t_{0}, \cdot\right) ; t_{0}\right]\right)
\end{gathered}
$$

Hence using (53) we conclude

$$
\begin{aligned}
\lim _{t \rightarrow t_{0}}\left\|u_{t}(t, \cdot)-u_{t}\left(t_{0}, \cdot\right)\right\|_{L^{2}(\Sigma)} & =0 \\
\lim _{t \rightarrow t_{0}}\left\|u(t, \cdot)-u\left(t_{0}, \cdot\right)\right\|_{H_{0}^{1}(\Sigma)} & =0
\end{aligned}
$$

So that $u$ is an element of $C\left([0, T] ; H_{0}^{1}(\Sigma)\right) \cap C^{1}\left(0, T ; L^{2}(\Sigma)\right)$ as required.

\section{Discussion}

In this section we relate our work to previous work in the area and discuss how Theorem 1 applies to several physical scenarios. We treat the case of spacetimes with cosmic strings and show that these spacetimes, despite having regions where the curvature behaves as a distribution, or in the case of dynamic cosmic strings can even develop curvature singularities, are $H^{1}$ wave regular. We end by discussing how the concept of $H^{1}$-wave regularity is related to the Strong Cosmic Censorship Conjecture.

The work in this paper extends that of Kay and Studer [15] who studied $L^{2}$ solutions of the wave equation in spacetimes with conical singularities. Our work generalises this in two ways, firstly we look at $H^{1}$ solutions and secondly we do not require the static or symmetry assumptions used in [15. A class of $H^{1}$ solutions were also considered by Ishibashi and Hasoya [7] who looked at finite energy solutions of the wave equation in a particular class of singular static spacetimes. Our work generalises this by removing the static assumption and allowing a wider class of spacetimes. An alternative approach to the study of conical singularities was initiated by Vickers and Wilson [16] using an approach based on generalised functions [17]. This was then extended to a wider class of singular spacetimes by Grant et al [18] who showed the existence of generalised solutions to the wave equation. The approach adopted in [16] and [18] involves regularising the equation by replacing the singular metric by a 1-parameter family of smooth metrics. The 1-parameter family of solutions of the corresponding wave equations then describes a generalised solution. The problem is then to show that such a solution is associated to a distributional solution of the wave equation. This is possible for the case of a cone but not for more general geometries. In our approach rather than regularising the equation we have looked at approximate solutions using the Galerkin method. The advantage of this is 
that rather than looking at a family of hyperbolic equations we are looking at a system of ODEs. By using the geometric conditions on the metric we have good analytic control over the solutions which enable us to show that these approximate solutions converge to a weak solution of the wave equation. The method we use follows that of Evans [11] but our results differ from his since we assume less regularity and as a result we are only able to show the existence of weak solutions.

\subsection{Spacetimes with Cosmic Strings}

Cosmic strings are topological defects that potentially formed during a phase transition in the early universe. Current observations put tight constrains on the dimensionless string tension $G \mu \leqslant 10^{-8}$ in Planck units where $c=1$, $G=m_{p l}^{-2}$ and $\mu$ is the mass per length [14]. Additionally, the effective thickness of a cosmic string is of the order $10^{-29} \mathrm{~cm}$. [19]. This extremely small width justifies what is called "the thin string limit". This is the metric around a static infinitely straight Nambu-Goto string lying along the z-axis satisfying Einstein's equations, which is "conical" in the plane transverse to the string with the line element given by

$$
d s^{2}=d t^{2}-d z^{2}-d \rho^{2}-(1-4 G \mu)^{2} \rho^{2} d \theta^{2}
$$

where $0 \leqslant \theta<2 \pi$.

By introducing a new angular coordinate $\tilde{\theta}=(1-4 G \mu) \theta$, the spacetime can be seen to be flat everywhere except at $\rho=0$ where there is an angular deficit of $2 \pi(1-A)$ with $A=(1-4 G \mu)$. We want to consider the region containing $\rho=0$ so we transform to Cartesian coordinates $(x, y)=(\rho \cos \theta, \rho \sin \theta)$ which are regular at $\rho=0$ and rewrite the line element as

$$
d s^{2}=d t^{2}-\frac{x^{2}+A^{2} y^{2}}{x^{2}+y^{2}} d x^{2}-\frac{2 x y\left(1-A^{2}\right)}{x^{2}+y^{2}} d x d y-\frac{y^{2}+A^{2} x^{2}}{x^{2}+y^{2}} d y^{2}-d z^{2}
$$

Notice that the metric has a direction dependant limit on the $z$-axis so fails to be $C^{0}$ at the axis although it remains bounded.

By direct inspection one can see that the metric is bounded everywhere, $N=1, \sqrt{\gamma}=A$ and given that $0<A \leqslant 1$ the uniform ellipticity condition is satisfied. These conditions imply that under a rescaling of the time coordinate the hypothesis of Theorem 1 is satisfied. Therefore, this spacetime is $H^{1}$-wave regular.

In fact, we can consider a time dependent generalisation of this metric given

$$
\begin{array}{r}
d s^{2}=d t^{2}-\frac{x^{2}+A^{2}(t) y^{2}}{x^{2}+y^{2}} d x^{2}-\frac{2 x y\left(1-A^{2}(t)\right)}{x^{2}+y^{2}} d x d y \\
-\frac{y^{2}+A^{2}(t) x^{2}}{x^{2}+y^{2}} d y^{2}-d z^{2}
\end{array}
$$


This spacetime represents a dynamical cosmic string with a time dependent deficit angle. The metric satisfies the conditions of the Theorem 1 as long as the angle deficit satisfies $0<A(t) \leqslant 1$ and the function $A(t)$ is $C^{1}$. Moreover, the dynamical cone can develop curvature singularities in contrast with the static cone [20].

Vickers [21, 22] showed that under certain conditions two-dimensional quasiregular singularities can be seen as generalised strings. Moreover, the strings are totally geodesic and only the normal directions to the string are degenerate. For timelike generalised cosmic strings this guarantees that the time derivatives are not problematic.

Finally, assuming that that the generalised cosmic string admits a $3+1$ splitting given by a family of $L^{\infty}$ Riemaniann metrics with suitable lapse and shift (see geometric conditions 1) and the angle deficit is chosen such that the uniform ellipticity condition is satisfied, we can conclude that generalised cosmic strings are $H^{1}$-wave regular.

Notice however that the spinning cosmic string metric given by

$$
d s^{2}=\left(d t^{2}+4 J d \theta\right)^{2}-d r^{2}-A^{2} r^{2} d \theta-d z^{2}
$$

does not satisfy the hypothesis of the theorem since $\beta^{i} \neq 0$ and there are no local coordinates containing a neighbourhood of the $z$-axis which make $\beta^{i}$ vanish.

It is also worth noting that the ability to look at solutions to the wave equation in spacetimes containing cosmic strings introduces the possibility of considering solutions to the wave equation on the Gott 2-string spacetime 23. This is an example of a spacetime which contains closed timelike curves and the Cauchy problem has been studied previously for a certain class of spacetimes with closed timelike curves [24]. A subsequent paper [25] includes a discussion of solutions to the wave equation in a 4-dimensional version of Misner space which has global properties similar to that of Gott space. However unlike the $H^{1}$ solutions we consider here the solutions considered in [25] are in $L_{l o c}^{2}$ which does not guarantee the local integrability of the energy-momentum tensor. 


\subsection{The Strong Cosmic Censorship Conjecture}

As mentioned in the introduction, the singularity theorems only establish geodesic incompleteness and not the reason for the incompleteness. In examples such as the Kerr and Reissner-Nordström spacetimes there is a loss of global hyperbolicity instead of a loss of regularity. Mathematically a spacetime region $\mathcal{N}$ is said to globally hyperbolic if the causality condition is satisfied and for any two points $p, q \in \mathcal{N}$ the causal diamond $J^{+}(p) \cap J^{-}(q)$ is compact and contained in $\mathcal{N}$ [26]. One demands this condition because it is sufficient to guarantee the global well-posedness of the wave equation and other physical fields [27]. Therefore, a spacetime that fails to be globally hyperbolic signals the possibility of a loss of predictability of the initial value problem of any field on it including the metric. While the maximal globally hyperbolic development is unique [1] one may extend the spacetime (even in a $C^{\infty}$ manner) in non-unique ways if one does not demand global hyperbolicity. The boundary of the original manifold in the extension is known as the Cauchy horizon. It is expected that under small perturbations the extensions are unstable and some loss of regularity will occur preventing the extension. This is part of the content of The Strong Cosmic Censorship Conjecture. In fact, Dafermos has shown that the outcome of the conjecture depends critically on the differentiability of the metric allowed in the extensions of the maximal Cauchy development [28].

As we noted in the introduction and showed in our results, a necessary condition for a singularity to be regarded as a strong gravitational singularity is that in any neighbourhood of it the evolution of the wave equation is not well posed. In this spirit, a well-motivated physical formulation of predictability may include the criteria of locally well-posedness of test field as a necessary condition. We therefore propose the following definition of "predictability".

Definition 3 An initial value set for Einstein's equations is said to be predictable if there is no nontrivial future extension of its maximal development such that the extension is $H^{1}$-wave regular.

Under this criteria the Kerr and Reissner-Nordström fail to be predictable as there exist (different) extensions of these spacetimes for which local wellposedness of the wave equation does not posses any difficulties. Nevertheless, as mention above, a loss of regularity is expected under small perturbations and a obstruction to $H^{1}$-wave regularity then must take place. This "generic" initial data then would be predictable.

Notice that one could also formulate a condition of predictability in terms of global-well posedness of the wave equation in $H^{1}$ rather than just local wellposedness. Under this criteria the Kerr and Reissner-Nordströmar spacetimes are predictable under certain conditions of the decay rates of the field at the event horizon which are crucial to the existence of an $H_{l o c}^{1}$ solution at the interior up to the Cauchy Horizon [29, 30]. More strikingly, is the fact 
that in the case of extremal Reissner-Nordström or Reissner-Nordström-DeSitter (RN-DS) one can prove that such finite energy solutions exist [31, 32. In fact, these works support recent evidence that the Strong Cosmic Censorship may not hold in these scenarios under the criteria of the regularity of $C^{0}$ metrics and $L^{2}$-Christoffel symbols.

\section{Acknowledgements}

The authors would like to thank CONACyT for supporting this work through a CONACyT Graduate Fellowship. 


\section{References}

[1] S. W. Hawking, G. F. R. Ellis, The large scale structure of space-time Cambridge University Press, (1974).

[2] M. Kunzinger, R. Steinbauer, M. Stojkovic, J. Vickers 2014 Gen. Relativ. Gravit. 461738

[3] P. T. Chruściel, J. D. E. Grant 2012 Class. Quantum Grav. 29145001

[4] T. J. R. Hughes, T. Kato, J. E. Marsden 1977 Archive for Rational Mechanics and Analysis 633

[5] S. Klainerman, I. Rodnianski, J. Szeftel 2012 ArXiv.1204.1767

[6] C. J. S. Clarke 1998 Class. Quantum Grav. 15975

[7] A. Ishibashi, A. Hosoya 1999 Phy. Rev. D 60, 104028

[8] Y. Sanchez Sanchez 2015 Gen. Relativ. Gravit. 4780

[9] Y. Sanchez Sanchez, J. A. Vickers 2015 ArXiv:1507.06463

[10] R. Wald, Quantum field Theory in Curved Spacetime and Black Hole Thermodynamics Chicago University Press, (1994)

[11] L. Evans Partial Differential Equations American Mathematical Society (1998)

[12] J. Hunter Lecture Notes https://www.math.ucdavis.edu/ hunter/ pdes/pde_notes.pdf

[13] M. B. Hindmarsh and T. W. B. Kibble 1995 Rep. Prog. Phys. 58477

[14] LIGO Scientific Collaboration and Virgo Collaboration 2014 Phys. Rev. Lett. 11212

[15] B. S. Kay, U. M. Studer 1991 Communications in Mathematical Physics 139, 103

[16] J. A. Vickers, J. P. Wilson 2000 Class. Quantum Grav. 17 1333-1360

[17] R. Steinbauer, J.A. Vickers 2008 Class. Quantum Grav. 266

[18] J.D.E. Grant, E. Mayerhofer, and R. Steinbauer, 2009 Comm. Math. Phys. 285 399-4

[19] M. R. Anderson, The Mathematical Theory of Cosmic Strings: Cosmic Strings in the Wire Approximation CRC Press, (2002).

[20] J. P. Wilson PhD Thesis University of Southampton (1997) 
[21] J. A. Vickers 1990 Class. Quantum Grav. 7731

[22] J. A. Vickers 1987 Class. Quantum Grav. 41

[23] J. R. Gott 1991 Phys. Rev. Lett. 66, 1126

[24] J. L. Friedman and M. S. Morris 1991 Phys. Rev. Lett. 66, 401

[25] J. L. Friedman and M. S. Morris 1991 Comm. Math. Phys. 186495

[26] A. N. Bernal, M. Sánchez, 2007 Class. Quantum Grav. 24745

[27] C. Bär, N. Ginoux, F. Pfäffle Wave Equations on Lorentzian Manifolds and Quantization ESI (2007).

[28] M. Dafermos 2003 Annals of Mathematics 158875

[29] J. Luk, J. Sbierski 2015 ArXiv:1512.08259

[30] A. Franzen 2014 ArXiv:1407.7093

[31] S. Aretakis 2011 Comm. Math. Phys. 30717

[32] J. L. Costa, A. Franzen 2016 ArXiv:160\%.01018 